\title{
Quantitative Nanomechanical Properties of Multilayer Films Made of Polysaccharides through Spray Assisted Layer-by-Layer Assembly
}

\author{
Miryam Criado, ${ }^{\dagger}$ Esther Rebollar, ${ }^{\ddagger}$ Aurora Nogales, ${ }^{\S}$ Tiberio A. Ezquerra, ${ }^{\S}$ Fouzia Boulmedais, ${ }^{\|}$ \\ Carmen Mijangos, ${ }^{\dagger}$ and Rebeca Hernández ${ }^{*},{ }^{\dagger}$ \\ ${ }^{\dagger}$ Instituto de Ciencia y Tecnología de Polímeros, ICTP-CSIC, Juan de la Cierva, 3, 28006 Madrid, Spain
${ }^{\ddagger}$ Instituto de Química Física Rocasolano, IQFR-CSIC, Serrano 119, 28006 Madrid, Spain
${ }^{\S}$ Instituto de Estructura de la Materia, IEM-CSIC, Serrano 121, 28006 Madrid, Spain \\ "Institut Charles Sadron, Centre National de la Recherche Scientifique, Université de Strasbourg, 23 rue du Loess, 67034 Cedex 2 \\ Strasbourg, France
}

ABSTRACT: Nanomechanical properties of alginate/chitosan (Alg/Chi) multilayer films, obtained through spray assisted layer-by-layer assembly, were studied by means of PeakForce quantitative nanomechanical mapping atomic force microscopy (PF-QNM AFM). Prepared at two different alginate concentrations ( 1.0 and $2.5 \mathrm{mg} / \mathrm{mL}$ ) and a fixed chitosan concentration $(1.0 \mathrm{mg} / \mathrm{mL}), \mathrm{Alg} / \mathrm{Chi}$ films have an exponential growth in thickness with a transition to a linear growth toward a plateau by increasing the number of deposited bilayers. Height, elastic modulus, deformation, and
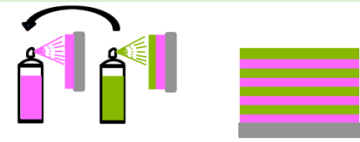

3 um
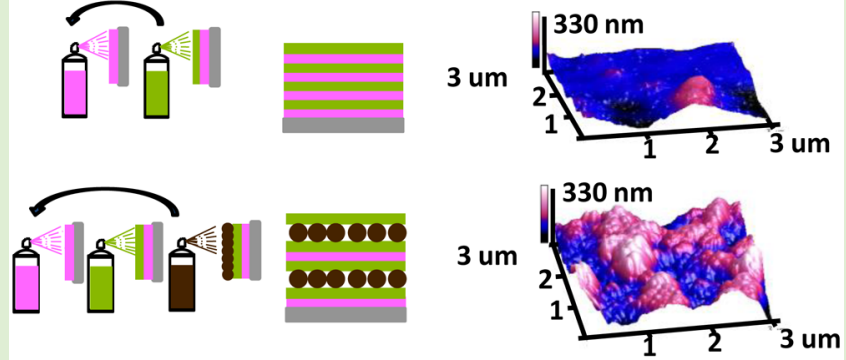
the number of deposited bilayers. The elastic modulus of $\mathrm{Alg} / \mathrm{Chi}$ films was found to be related to the mechanism of growth in contrast to the adhesion and deformation. A comparison of the nanomechanical properties obtained for non-cross-linked and thermally cross-linked $\mathrm{Alg} / \mathrm{Chi}$ films revealed an increase of the elastic modulus after cross-linking regardless alginate concentration. The incorporation of iron oxide nanoparticles (NPs), during the spray preparation of the films, gave rise to nanocomposite $\mathrm{Alg} / \mathrm{Chi}$ films with increased elastic moduli with the number of incorporated NPs layers. Deformation maps of the films strongly suggested the presence of empty spaces associated with the method of preparation. Finally, adhesion measurements point out to a significant role of NPs on the increase of the adhesion values found for nanocomposite films.

\section{INTRODUCTION}

Over the past few years, multilayer polymer films and nanocomposite films, obtained through their combination with nanoparticles, have drawn a lot of attention for the development of several applications in organic microelectronics, as biosensors or scaffolds for tissue engineering. ${ }^{1,2}$ The control of the mechanical properties of these films is specifically attractive in biomaterials area where the mechanical properties of polymer films allow to modulate cellular behaviors regarding adhesion, proliferation and cell differentiation. ${ }^{3-5}$ In this context, the layer-by-layer ( $\mathrm{LbL}$ ) assembly of films based on polysaccharides, which are inherently biocompatible, constitutes a powerful route for the production of stratified polymer films employed in biomedical applications. In particular, multilayer films based on chitosan (Chi) and alginate (Alg), two polysaccharides extracted from shells of crustacean or fungal mycelia and brown seaweed, respectively, ${ }^{6,7}$ have drawn a lot of attention in this field as patches for tissue engineering 8,9 and membranes for drug delivery. ${ }^{10,11}$

Until now, most of the mechanical studies performed on $\mathrm{LbL}$ polymer multilayer and nanocomposite multilayer films have been accomplished by macroscopic stress-strain experiments.
Focusing on $\mathrm{Alg} / \mathrm{Chi}$ multilayer films, measurements of their mechanical strength using the bulge test have revealed an increase in the elastic modulus with the film thickness for films in the range of tens of nanometers. ${ }^{8}$ Very recently, it was shown that the incorporation of magnetic nanoparticles within freestanding Alg/Chi multilayer films gave rise to an increase in the elastic moduli measured in the wet state by stress-strain measurements. ${ }^{12}$ Probe-based indentation is a versatile method to study local mechanical properties of materials from the nanoscale to the macroscale. ${ }^{13-15}$ Within this method, nanoindenter and atomic force microscopy (AFM) are the most used techniques. ${ }^{16}$ An early study reported the employment of AFM indentation, with $2.5 \mu \mathrm{m}$ radius sphere, to determine the mechanical properties of hydrated Poly(Llysine)/hyaluronic acid LbL films in the micron range either native or cross-linked and supported by an underlying glass coverslip. ${ }^{17}$ However, this technique presents some drawbacks such as poor lateral resolution and the large time required to

Received: September 29, 2016

Revised: November 7, 2016

Published: December 1, 2016 
Scheme 1. Schematic Diagram of the Sequential Spraying Process Followed for the Preparation of Nanocomposite (Alg/Chi) ${ }_{n} /$ $(\mathrm{NPs} / \mathrm{Chi})_{m}$ films

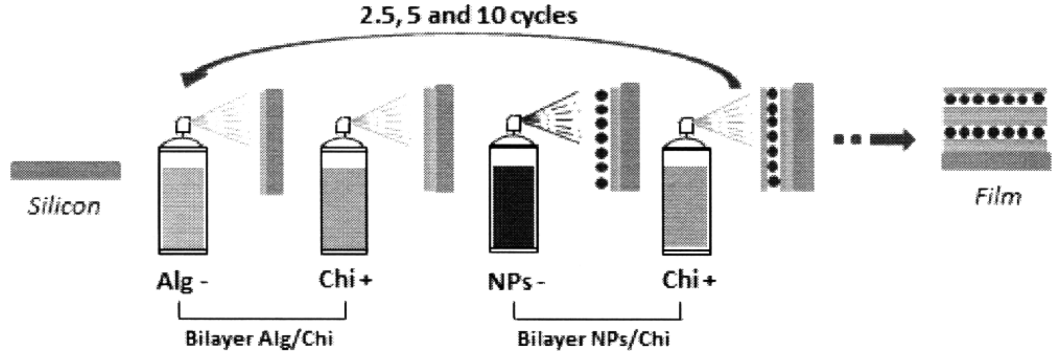

obtain the quantitative mechanical mapping of the surface. ${ }^{13,15}$ To solve these problems, two AFM modes for mapping mechanical properties have been introduced, HarmoniX and PeakForce (PF) quantitative nanomechanical mapping (QNM). ${ }^{13,15}$ These modes use the Derjaguin-Muller-Toporov (DMT) model $^{18}$ to obtain the elastic modulus from the force-indentation curves. It is important to note that the employment of regular sharp probes may cause overestimation of the film modulus by the DMT method. An approach would be using special probes, such as colloidal ones for example, that would allow increasing the applied force without producing large penetration. However, in that case, lateral resolution would be compromised. ${ }^{13}$

There are only few studies aimed to determine the nanomechanical properties of LbL multilayer films by means of (PF-QNM) AFM, mainly focused on determining the effect of the number of deposited layers on the elastic moduli on LbL polymer films and the effect of the stiff underlying substrate ${ }^{19,20}$ and, none of them, deal with the determination of the nanomechanical properties of films fully fabricated from polysaccharides. In this paper, we aim to determine the nanomechanical properties (elastic moduli, deformation, and adhesion values) of alginate/chitosan multilayer films. To achieve this aim, we not only considered the effect of the number of deposited layers on the resulting mechanical properties but we also considered the effect of cross-linking and the effect of the incorporation of nanoparticles to obtain nanocomposite films during the buildup of the films. All of these parameters are known to influence the performance of these materials in biomedical applications. Specifically, nanocomposite Alg/Chi films can be employed as dermal pads for local magnetic hyperthermia. ${ }^{21}$ Samples were prepared through a spray assisted LbL method which allowed us to obtain films in a wide range of thicknesses (from nano to the micron size) with considerable saving of time with respect to samples prepared through immersion procedures. Finally, we aimed to determine the mechanism of growth of $\mathrm{Alg} / \mathrm{Chi}$ films and correlate it to their nanomechanical properties and hence to the structure generated during the spray buildup.

\section{EXPERIMENTAL SECTION}

Materials. Low molecular weight Chitosan (Chi) was supplied by Aldrich (448869, lot SLBG1673V). According to the fabricant, viscosity was $20-300 \mathrm{cps}$ ( $1 \mathrm{wt} \%$ in acetic acid, $25{ }^{\circ} \mathrm{C}$, Brookfield). Purified using the procedure described by Signini and Campana Filho, ${ }^{22}$ Chi was dissolved in acetic acid $1 \% \mathrm{w} / \mathrm{v}$ with magnetic stirring for $22 \mathrm{~h}$ and filtered through filter paper. Then, precipitation of Chi was obtained by addition of $6.25 \mathrm{M} \mathrm{NaOH}$ drop by drop. After that, Chi was filtered and washed with distilled water until neutral $\mathrm{pH}$. At the end, it was rinsed with ethanol and dried in an oven at $55^{\circ} \mathrm{C}$ for $17 \mathrm{~h}$. The deacetylation degree (DD) of Chi, determined by $\mathrm{RMN}-{ }^{1} \mathrm{H}$ at $70{ }^{\circ} \mathrm{C}$ using 2 wt $\% \mathrm{CD}_{3} \mathrm{COOD} / \mathrm{D}_{2} \mathrm{O}$ as solvent, ${ }^{23}$ was of $81 \%$. Molecular weight $\left(M_{\mathrm{w}}\right)$ of Chi, determined by capillary viscosimetry at $25{ }^{\circ} \mathrm{C}$ using as solvent acetic acid $0.3 \mathrm{M}$ /sodium acetate $0.2 \mathrm{M}$ and applying the equation of Mark-Houwink $\left(k=74 \times 10^{-5} \mathrm{dL} / \mathrm{g}, a=\right.$ $0.76)$, ${ }^{24}$ was of $67000 \mathrm{Da}$. Sodium alginate (Alg) was supplied by Sigma-Aldrich (A2158, lot 090M0092V). According to the fabricant, its viscosity was $136 \mathrm{cps}\left(2 \% \mathrm{w} / \mathrm{v}\right.$ in water at $\left.25^{\circ} \mathrm{C}\right)$. Molecular weight $\left(M_{\mathrm{w}}\right)$ of $\mathrm{Alg}$, determined by capillary viscosimetry at $25^{\circ} \mathrm{C}$ using as solvent sodium chloride $0.1 \mathrm{M}$ and applying the equation of MarkHouwink $\left(k=2 \times 10^{-5} \mathrm{dL} / \mathrm{g}, a=1.0\right),{ }^{25}$ was $166000 \mathrm{Da}$. Poly(ethylenimine) (PEI), with a molecular weight $\left(M_{\mathrm{w}}\right)$ of 25000 , and acetic acid were supplied by Aldrich and used as received. Sodium acetate anhydrous was supplied by Panreac and chloride acid by VWR. A lab-made aqueous ferrofluid containing the iron oxide nanoparticles (NPs) $(8 \mathrm{mg} / \mathrm{mL})$ was employed to prepare nanocomposite $\mathrm{Alg} / \mathrm{Chi}$ films. $^{26}$

Multilayer Films Preparation. All films were built on silicon wafers $(20 \times 40 \mathrm{~mm}$, Siegert $)$ previously cleaned with piranha solution (3:1 $\left.\mathrm{H}_{2} \mathrm{SO}_{4} / \mathrm{H}_{2} \mathrm{O}_{2}\right)$ for $10 \mathrm{~min}$ and rinsed extensively with water. As the silicon substrate is negatively charged, a first layer of PEI $(1 \mathrm{mg} /$ $\mathrm{mL}$ ) was deposited providing a positively charged homogeneous substrate. For that purpose, substrates were immersed in a solution of PEI for $5 \mathrm{~min}$, rinsed in a solution of distilled water for $2 \mathrm{~min}$ and subsequently dried. During the spraying process, the substrate was inclined $45^{\circ}$ with respect to the vertical to allow the drainage of the solution. The multilayer films were built by a sequential spraying of either Alg or NPs (negatively charged compound) and Chi (polycation). A fixed Chi concentration $(1 \mathrm{mg} / \mathrm{mL})$ and three different concentrations of Alg solution were used 1,2.5, and $5 \mathrm{mg} /$ $\mathrm{mL}$. The concentration of Chi was chosen based on the fact that higher concentrations tend to clog the spray. Three different LbL systems were prepared: (i) $\mathrm{Alg} / \mathrm{Chi}$ films, (ii) cross-linked $\mathrm{Alg} / \mathrm{Chi}$ films, and (iii) nanocomposite $\mathrm{Alg} / \mathrm{Chi}$ films. For the preparation of $\mathrm{Alg} / \mathrm{Chi}$ films, Alg solution was sprayed onto the substrate for $5 \mathrm{~s}$. After a waiting time of $15 \mathrm{~s}$, Chi solution was sprayed for $5 \mathrm{~s}$. After an additional waiting time of $15 \mathrm{~s}$, the cycle was repeated until obtaining the desired number of layers. Samples were denoted as $(\operatorname{Alg} x / \mathrm{Chi})_{n}$ where $n$ stands for the number of bilayers $(n=5,10,20$ or 40$)$, and $x$ refers to Alg concentration $(x=1,2.5$, or $5 \mathrm{mg} / \mathrm{mL})$. The second type of samples was obtained by cross-linking of the previously deposited $\mathrm{Alg} / \mathrm{Chi}$ films at $120{ }^{\circ} \mathrm{C}$ for $2 \mathrm{~h}$. The third series of samples was obtained by spraying a bilayer of alginate and chitosan followed by a bilayer of a lab-made ferrofluid containing the iron oxide NPs and a chitosan layer (Scheme 1). In this case, a fixed alginate solution with a concentration of $2.5 \mathrm{mg} / \mathrm{mL}$ was used and each layer was sprayed for 5 s. Three nanocomposite films were obtained by applying $2.5,5$, and 10 cycles and samples were denoted as $(\mathrm{Alg} / \mathrm{Chi})_{n} /(\mathrm{NPs} / \mathrm{Chi})_{m}$, where $n$ stands for the number of $\mathrm{Alg} / \mathrm{Chi}$ bilayers $(n=3,5$ and 10) and $m$ stands for the number of NPs/Chi bilayers $(m=2,5$ and 10). The spray process was carried out manually. The repeatability is guaranteed because the air brush is placed at a fixed distance in relation to the sample $(100 \mathrm{~mm})$ and the spray of the polymer solutions is controlled by the air pressure $(150 \mathrm{bar})$ and the deposition time $(5 \mathrm{~s})$.

Multilayer Films Characterization. Measurements of the film thickness were carried out with a PLASMOS SD 2300 ellipsometer 
operating at the single wavelength of $632.8 \mathrm{~nm}$ and a constant angle of $70^{\circ}$. Due to the inherent limit of ellipsometry to simultaneously determine the refractive index and the film thickness for very thin films, the refractive index of all films was assumed to be constant at 1.465 . $^{27}$ While this procedure will lead to slightly incorrect absolute values, it allows for the quick and precise determination of relative film thicknesses. For each substrate studied, 10 different thickness measurements were randomly taken on different film regions over an area of a few square centimeters of the film surface. The cross section of films was observed by scanning electron microscopy (SEM), using a Hitachi SU 8000 microscope operating at $0.8 \mathrm{kV}$. The average thickness was determined by measuring the width of the cross section in the SEM micrographs in five different points.

Atomic Force Microscopy (AFM) Nanoindentation Measurements. The topography, surface roughness and mechanical properties of films were evaluated using a Bruker Multimode 8, with a Nanoscope $\mathrm{V}$ controller, by the PeakForce Quantitative Nanomechanical Mapping (PF-QNM) method. All measurements were performed in air, at room temperature, on films supported on silicon wafers. Samples (Algx/ $\mathrm{Chi})_{\mathrm{n}}$ were scanned over square regions of $2 \mu \mathrm{m}$ size and nanocomposite $(\mathrm{Alg} / \mathrm{Chi})_{n} /(\mathrm{NPs} / \mathrm{Chi})_{m}$ films over square regions of $1 \mu \mathrm{m}$ size. The average roughness $\left(R_{\mathrm{a}}\right)$ was calculated from the height images in four different square points of $1 \mu \mathrm{m} \times 1 \mu \mathrm{m}$. All quantitative measurements were carried by using different probes depending on the actual mechanical properties of every sample. In particular, we used TAP150 and RTESPA525 probes (Bruker). Cantilever spring constants for each probe were measured by the thermal tune method and found to be around $1-5 \mathrm{~N} / \mathrm{m}$ for TAP 150 employed for the measurements of films without nanoparticles and 50-100 N/m for RTESPA525 employed for the measurements of nanocomposite films. Every tip radius was calibrated against a polystyrene standard of known elastic modulus (Bruker), considering the DMT model ${ }^{18}$ through eq 1 :

$$
E_{\mathrm{r}}=\frac{3\left(F_{\text {tip }}-F_{\text {adh }}\right)}{4 \sqrt{R d^{3}}}
$$

where $E_{\mathrm{r}}$ is the reduced Young modulus. For tips modulus, $F_{\text {tip }}$ is the force on the tip, $F_{\text {adh }}$ is the adhesive force between the AFM tip and the sample, $R$ is the AFM tip radius, and $d$ is the penetration depth. Provided that the tip Young modulus is much higher than the sample modulus, the reduced modulus and the Young modulus are related through eq 2

$$
E_{\mathrm{s}}=\frac{E_{\mathrm{r}}}{1-\nu_{\mathrm{s}}^{2}}
$$

where $\nu$ is the Poisson's ratio value.

The precise experimental procedure was the following: An initial $F_{\text {tip }}$ was set, and an initial estimation of the tip radius, based on the provider specifications was considered. The $F_{\text {tip }}$ was chosen to produce a deformation depth of about $5 \mathrm{~nm}$. Under these experimental conditions, $F_{\text {adh }}$ can be neglected. With this, an experiment on the polystyrene standard was carried out. The tip radius value was modified systematically until the measured elastic modulus of the reference matched the one provided by the provider. The obtained value for the tip radius was typically around $10 \mathrm{~nm}$. The same sample was measured with different tips, after careful calibration for tip radius, for cantilever deflection sensitivity and also at different times. In addition, the $z$ distance in the piezo is regularly calibrated using a grating of known depth.

PF-QNM is a contact AFM protocol which is based on the forcevolume method. Here, force-distance curves are collected by nanoindentation of the sample in a point-by-point fashion. In this method, the piezo-scanner is oscillated at $2 \mathrm{kHz}$ while the probe remains at rest. This fact enables a high speed and simultaneous capture of force-distance curves and topographic images. ${ }^{28}$ The maximum force (peak force) is controlled at each pixel to obtain force-distance curves which are then used as feedback signal.

Figure 1 shows an example of the PF-QNM force-distance curve corresponding to the sample (Alg2.5/Chi) $)_{10}$. The $Z$-axis reflects the

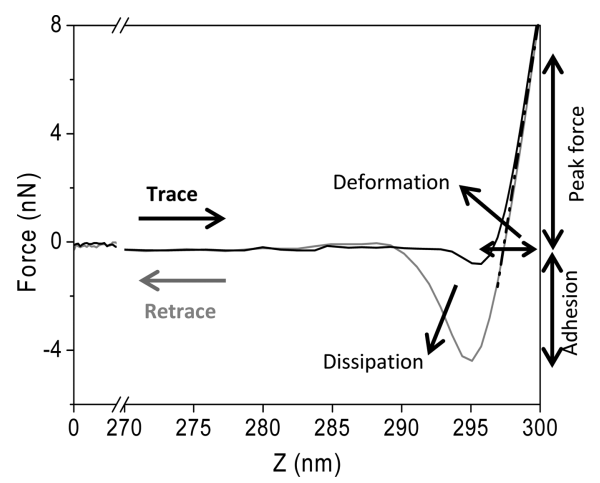

Figure 1. Force-distance curve of the sample (Alg2.5/Chi) $)_{10}$ while approaching the surface (trace) and withdrawal (retrace). The dashed line corresponds to fitting of the experimental results to the DMT model.

distance ramped by the piezo-scanner in the vertical direction. The trace curve is obtained when the tip is approaching the surface of the sample and the retrace curve when the tip is withdrawing from it. As it can be observed, an attractive force appears when the tip is approaching the sample followed by an elastic regime in which the force is proportional to the deformation. The adhesion force is related to the minimum of the retrace force-distance curve (upon withdrawal) and the dissipation is the area between the trace and retrace of the force-distance curves.

Analysis of the force-distance curves is automatically performed by a software and allows to extract the height, elastic modulus, adhesion force, deformation and dissipation simultaneously by application of the DMT model. The employment of the DMT model is justified by the fact that the chosen regime of applied forces allows us to study the nanomechanical response of the bilayers in the elastic regime, i.e., without permanent deformation of the sample's surface. The Young's modulus is obtained by fitting the retrace curve using eqs 1 and 2 and a Poisson's ratio of 0.3 . $^{18}$ Note in Figure 1 that the part of the forcedistance curve used to estimate the DMT modulus superimposed in both loading and unloading regime. The DMT Young's Modulus obtained from the fitting was $2.9 \mathrm{GPa}$ which is in good agreement with the experimental value given by the software for sample (Alg2.5/ Chi) ${ }_{10}$ as shown in the Results and Discussion section.

\section{RESULTS AND DISCUSSION}

Mechanism of Growing of Alg/Chi Films Obtained through Spray Assisted LbL. In order to optimize the conditions of buildup via spray assisted $\mathrm{LbL},(\operatorname{Alg} x / \mathrm{Chi})_{n}$ multilayer films were prepared with different concentrations of Alg solution keeping the concentration of Chi solution fixed at $1.0 \mathrm{mg} / \mathrm{mL}$. Figure 2 shows the evolution of the thickness of $(\operatorname{Alg} x / \mathrm{Chi})_{n}$ films as a function of the number of bilayers for different Alg concentrations, where $n$ stands for the number of bilayers and $x$ refers to $\mathrm{Alg}$ concentration $(1,2.5$, and $5 \mathrm{mg} /$ $\mathrm{mL}$ ).

As it can be observed, the thickness of (Algx/Chi) multilayer films increases linearly with the number of bilayers, up to 5 bilayers, with a slope that increases with the concentration of Alg solution. Alg1/Chi film grows at a rate of $4 \mathrm{~nm} /$ layer whereas Alg2.5/Chi and Alg5/Chi films grow at a similar rate of $20 \mathrm{~nm}$ /layer. Previous reports have shown a linear increase of the thickness of $\mathrm{Alg} / \mathrm{Chi}$ films with the number of layers when prepared by LbL dipping at a $3 \mathrm{~nm} /$ layer rate which corresponds to a total thickness of $\sim 35 \mathrm{~nm}$ for $\mathrm{Alg}$ and Chi concentrations of $0.5 \mathrm{mg} / \mathrm{mL}$ at $\mathrm{pH} \mathrm{5.5.}{ }^{29}$ The growing of the film with the number of bilayers for the two highest concentrations of $\mathrm{Alg}, 2.5$ and $5.0 \mathrm{mg} / \mathrm{mL}$, is similar 


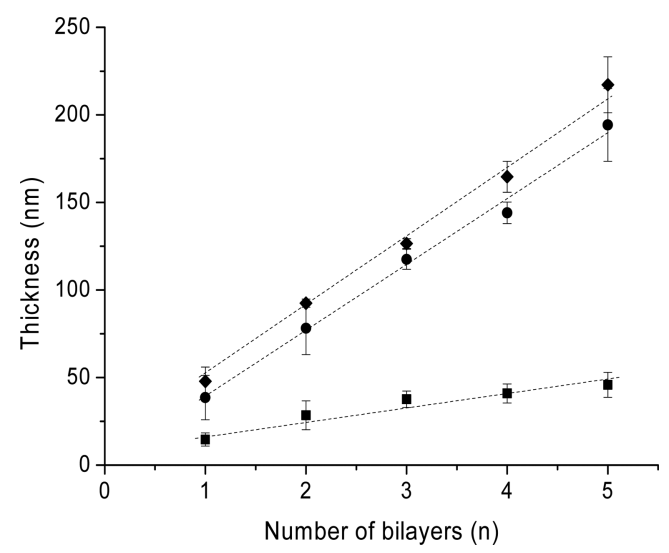

Figure 2. Evolution of $(\operatorname{Alg} x / C h i)_{n}$ film thickness, measured by ellipsometry, vs the number of bilayers. The films were obtained by spray assisted LbL with Alg concentration at $x=1.0(\boldsymbol{\square}), x=2.5(\bullet)$, and $x=5.0 \mathrm{mg} / \mathrm{mL}(\bullet)$. Dashed lines are a guide to the eye.

and much higher than that obtained for $1.0 \mathrm{mg} / \mathrm{mL}$ of alginate. It is worth mentioning that using $5.0 \mathrm{mg} / \mathrm{mL}$ Alg solution tends to clog the spray. Therefore, this concentration was discarded for the next experiments. So, the following experiments are focused on $(\operatorname{Alg} x / \mathrm{Chi})_{\mathrm{n}}$ films prepared with $x=1.0$ and 2.5 $\mathrm{mg} / \mathrm{mL}$.

Figure 3 shows the evolution of the thickness of $(\mathrm{Alg} 1 / \mathrm{Chi})_{n}$ and $(\mathrm{Alg} 2.5 / \mathrm{Chi})_{n}$ films as a function of the number of bilayers,

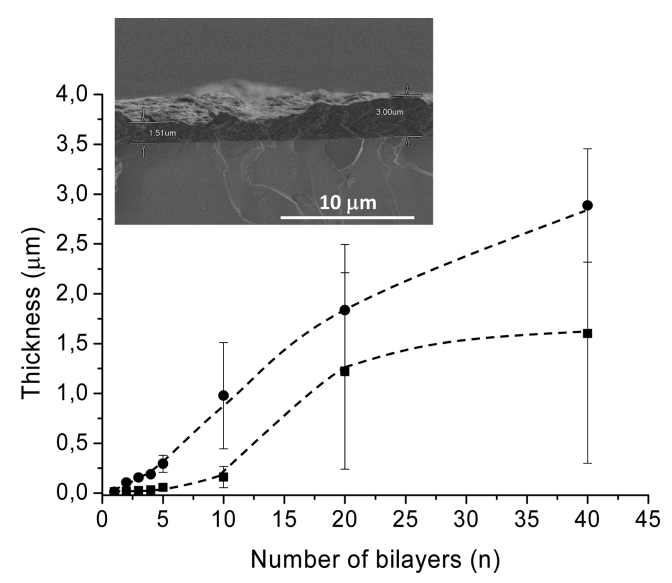

Figure 3. (a) Evolution of $(\mathrm{Alg} 1 / \mathrm{Chi})_{n}(\mathbf{\square})$ and $(\mathrm{Alg} 2.5 / \mathrm{Chi})_{n}(\mathbf{\bullet})$ film thickness, measured by SEM, vs number of bilayers. Dashed lines are a guide to the eye. The inset of the figure is a representative SEM micrograph corresponding to the cross section of a film (Alg2.5/ Chi) $)_{20}$.

up to $n=40$. The thickness of films with $n=10,20$, and 40 bilayers was determined by SEM due to the ellipsometry restrictions to determine film thicknesses of more than 8 bilayers. In order to visualize the cross section of Alg/Chi films, a representative SEM micrograph $(\mathrm{Alg} 2.5 / \mathrm{Chi})_{20}$ has been included in the inset of Figure 3. It can be observed that the thickness increases linearly up to 5 bilayers, exponentially from 5 to 20 deposited bilayers and then slightly increases toward a plateau.

Previous studies have shown that the multilayer growth regime is related to the internal structure of the multilayer film. ${ }^{19,30}$ For polymer films comprising several layers, the growth profile typically proceeds either linearly or exponen- tially. Exponential film growth is currently understood to arise from the diffusion of adsorbed species into and out of the multilayer film. In this process, macromolecules first adsorb from solution to the exposed surface of the film via attractive intermolecular interactions, then they diffuse through the bulk toward the substrate. Diffusion from the bulk of the film toward the film surface also occurs during this process. In the case of $(\operatorname{Alg} x / C h i)_{n}$ films reported in this study, the exponential growth could be due to the diffusion of chitosan "in" and "out" of the film during the deposition process. The diffusion of chitosan during the buildup process in different polyelectrolytes systems has been proved for chitosan/hyaluronic acid ${ }^{31}$ and chitosan/heparin ${ }^{32}$ up to 10 bilayers. The change of growth from exponential to linear at a number of bilayers higher than 20 might be due to the gradual rearrangement of the polymer chains in the film which makes the film less penetrable for the diffusion process, so that the growth becomes linear with the number of deposited layers when the time allowed for deposition is constant. ${ }^{33}$

Effect of Thermal Cross-Linking and Number of Bilayers on the Nanomechanical Properties. To characterize the nanomechanical properties of $\mathrm{Alg} / \mathrm{Chi} \mathrm{LbL}$ films by means of (PF-QNM) AFM, the number of bilayers was varied and the effect of the cross-linking was studied. $(\mathrm{Alg} 2.5 / \mathrm{Chi})_{n}$ films were subjected to a thermal treatment of $120^{\circ} \mathrm{C}$ during 2 $\mathrm{h}$ in order to induce cross-linking through formation of amide bonds between the amine groups of chitosan and the carboxylic groups from alginate. ${ }^{34}$ The average roughness $\left(R_{\mathrm{a}}\right)$ of noncross-linked and cross-linked $(\mathrm{Alg} 2.5 / \mathrm{Chi})_{n}$ films is shown in Table 1 as a function of the number of bilayers.

Table 1. Evolution of Average Roughness $\left(R_{\mathrm{a}}\right)$ of NonCross-Linked and Cross-Linked (Alg2.5/Chi) ${ }_{n}$ Films as a Function of the Number of Bilayers $(n)$

\begin{tabular}{ccc} 
& \multicolumn{2}{c}{ avg roughness $R_{\mathrm{a}}(\mathrm{nm})$} \\
\cline { 2 - 3 } no. of bilayers, $n$ & (Alg2.5/Chi) films & cross-linked (Alg2.5/Chi) films \\
5 & $8.9 \pm 3.6$ & $4.8 \pm 3.6$ \\
10 & $17.1 \pm 3.4$ & $3.8 \pm 0.6$ \\
20 & $29.8 \pm 11.9$ & $22.3 \pm 8.9$ \\
40 & $49.0 \pm 17.5$ & $18.5 \pm 0.5$ \\
\hline
\end{tabular}

Note that, in the case of non-cross-linked (Alg2.5/Chi) films, $R_{\mathrm{a}}$ increases from $8.9 \pm 3.6$ to $49.0 \pm 17.5 \mathrm{~nm}$ when the number of bilayers increases from 5 to 40 . This might be attributed to the self-assembly of opposite charges between alginate and chitosan that gives rise to their aggregation resulting in rougher surfaces as the number of layers increases. ${ }^{35}$ The $R_{\mathrm{a}}$ values obtained for cross-linked films are lower than those corresponding to native $(\mathrm{Alg} 2.5 / \mathrm{Chi})_{n}$ films in agreement with previous reports in which it was demonstrated that, cross-linking of multilayer films does not significantly change the thickness in the dry state, giving rise to more uniform films due to the reorganization of the film organization. ${ }^{11}$ Notice that $R_{\mathrm{a}}$ values obtained after crosslinking do not follow a clear trend with the deposited number of layers probably due to the fact that $R_{\mathrm{a}}$ arises during the process of the preparation of the films before the cross-linking step.

Representative (PF-QNM) AFM images corresponding to $(\mathrm{Alg} 2.5 / \mathrm{Chi})_{20}$ films before and after thermal cross-linking are shown in Figure 4. A comparison of the AFM height images and those corresponding to the mechanical properties (elastic 

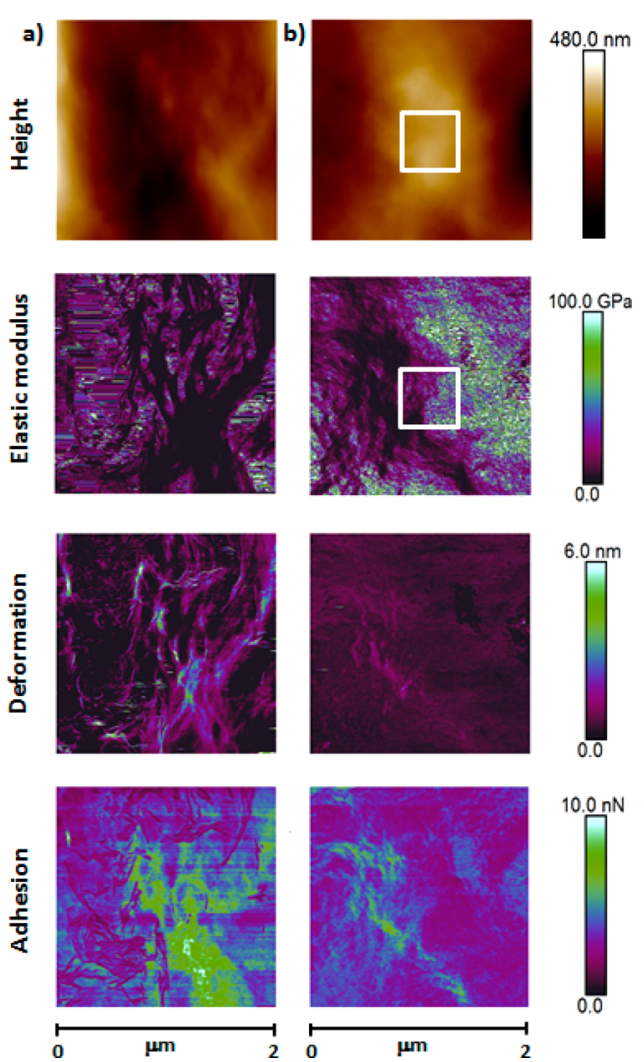

Figure 4. PF-QNM AFM images $(2 \mu \mathrm{m} \times 2 \mu \mathrm{m})$ of height, elastic modulus, deformation, and adhesion corresponding to $(\mathrm{Alg} 2.5 / \mathrm{Chi})_{20}$ films (a) before and (b) after cross-linking. For the sake of discussion, the white square $(500 \mathrm{~nm} \times 500 \mathrm{~nm})$ placed in the same position within the height AFM image and the elastic moduli AFM image indicates a small roughness region with differences in the elastic moduli.

modulus, deformation and adhesion) allows to observe that the topography of the samples is not associated with changes of the mechanical properties. This is more evident for cross-linked $(\mathrm{Alg} 2.5 / \mathrm{Chi})_{20}$ films. For easier visualization, a white square $(500 \mathrm{~nm} \times 500 \mathrm{~nm})$ was placed in the same position within the height AFM image and that corresponding to the determination of elastic modulus. As it can be observed, this region presents a homogeneous roughness whereas differences in the elastic moduli can be observed according to the different color scale. This result suggests the presence of heterogeneous regions which might be related to regions of different degree of cross-linking arising from differences on the rearrangement of the polymer layers after thermal cross-linking or even from inhomogeneous deposition of the polymers, regions with more polymer mass give rise to a higher number of cross-linking points. In addition, there is a correlation between AFM images corresponding to deformation and those corresponding to adhesion: regions that deform more are those that show a higher tip-adhesion force. This might be attributed to the establishment of more effective interactions, probably of van der Waals nature, between the tip and the polymer surface.

The mechanical properties (elastic modulus, deformation, and adhesion) were quantified at four different positions. The elastic modulus, the deformation and the adhesion of native $(\mathrm{Alg} x / \mathrm{Chi})_{n}$ as a function of the number of bilayers are shown in Figure 5a.
The elastic modulus slightly increases with the number of bilayers being $8.5 \pm 2.0 \mathrm{GPa}$ for 5 bilayers and $12.4 \pm 2.3 \mathrm{GPa}$ for 40 bilayers. The increase in the elastic moduli with the number of bilayers is more significant in the case of (Alg2.5/ Chi $)_{n}$ increasing from $0.8 \pm 0.1 \mathrm{GPa}$ to $11.8 \pm 4.3 \mathrm{GPa}$ from 5 to 40 bilayers, respectively. The elastic moduli obtained for $(\mathrm{Alg} 2.5 / \mathrm{Chi})_{n}$ films are lower than those corresponding to $(\mathrm{Alg} 1 / \mathrm{Chi})_{n}$ ones. For both alginate concentrations, the elastic moduli reach a plateau value of $\sim 12 \mathrm{GPa}$ at 40 bilayers. Note that this value is similar to those obtained by bulge tests carried out on free-standing $\mathrm{Alg} / \mathrm{Chi}$ films prepared by spray assisted $\mathrm{LbL}$ that showed that the elastic modulus of $35 \mathrm{~nm} \mathrm{Alg/Chi}$ films was $1.1 \pm 0.4 \mathrm{GPa}$ and increased to similar values for 75 and $114 \mathrm{~nm} \mathrm{Alg} /$ Chi films $(8.1 \pm 2.5 \mathrm{GPa}$ and $11.0 \pm 1.6 \mathrm{GPa}$ respectively). For these samples, a linear increase in the thickness is obtained with a single $\mathrm{Alg} / \mathrm{Chi}$ bilayer of approximately $3.3 \mathrm{~nm}$ thickness, suggesting that each polysaccharide layer constitutes an almost single molecular layer. ${ }^{8}$ In our study, spray assisted LbL of $(\mathrm{Alg} 1 / \mathrm{Chi})_{n}$ and $(\mathrm{Alg} 2.5 / \mathrm{Chi})_{n}$ films present a thickness of a single $\mathrm{Alg} / \mathrm{Chi}$ bilayer of $\sim 7.8 \mathrm{~nm}$ and $\sim 42.4 \mathrm{~nm}$, respectively and a plateau value for the elastic modulus of $12.4 \pm 2.3 \mathrm{GPa}$ and $11.8 \pm 4.3$ $\mathrm{GPa}$, respectively is obtained for $(\mathrm{Alg} x / \mathrm{Chi})_{n}$ films with thicknesses above $1 \mu \mathrm{m}$, i.e. above 20 bilayers. This fact may be attributed to the change in growth mechanism from exponential to linear that takes place above 20 bilayers as shown in Figure 3. Thus, it follows that the effective interaction established between $\mathrm{Alg}$ and $\mathrm{Chi}$ is weaker for sprayed $\mathrm{LbL}$ than for spin-coated LbL. We propose that the initial exponential growth observed in Figure 3 is controlled by the interdiffusion of both $\mathrm{Alg}$ and Chi leading to a significant increase of the elastic moduli with the increase of bilayers. Once the thickness of the sprayed LbL film reaches a certain value, the buildup changes from an exponential to linear growth due to a hindering of the diffusion process and the elastic modulus tends to a plateau value.

The deformation of Alg/Chi films is almost constant with the number of bilayers and only varies in a range between 0.5 and $1.5 \mathrm{~nm}$ due to the similar peak force used for all measurements. Similarly, adhesion values are independent of the number of deposited bilayers with values ranging between 1 and $4 \mathrm{nN}$. This is expected, as the adhesion is derived from the pull off force that is a measure of the attractive forces between the tip and outermost layers of the samples, i.e., the adhesion measurements are sensitive only to the surface and thus which should be similar for all the samples. Moreover, there are no differences in deformation and adhesion between films prepared at the two concentrations of Alg.

Now, we turn our attention to the effect of the cross-linking on the mechanical properties measured on $(\mathrm{Alg} 2.5 / \mathrm{Chi})_{n}$ films as a function of the number of bilayers $(n)$ (Figure $5 \mathrm{~b}$ ). For $n<$ 10 , the elastic moduli of cross-linked films are similar to those of the native ones. However, as the number of bilayers increases, the elastic moduli drastically increase to reach a maximum value of $\approx 47.4 \pm 3.8 \mathrm{GPa}$ at 40 bilayers. In addition, the deformation values of cross-linked $(\mathrm{Alg} 2.5 / \mathrm{Chi})_{n}$ films remain below $0.9 \mathrm{~nm}$ regardless the number of bilayers. Adhesion values are on the same range as those encountered for native films with the exception of $(\mathrm{Alg} 2.5 / \mathrm{Chi})_{5}$ film for which the adhesion force increases to $6 \mathrm{nN}$. Although no conclusive explanation for this effect exists, we can speculate about a lower chemical heterogeneity for the cross-linked sample at low number of bilayers arising from a lower degree of 

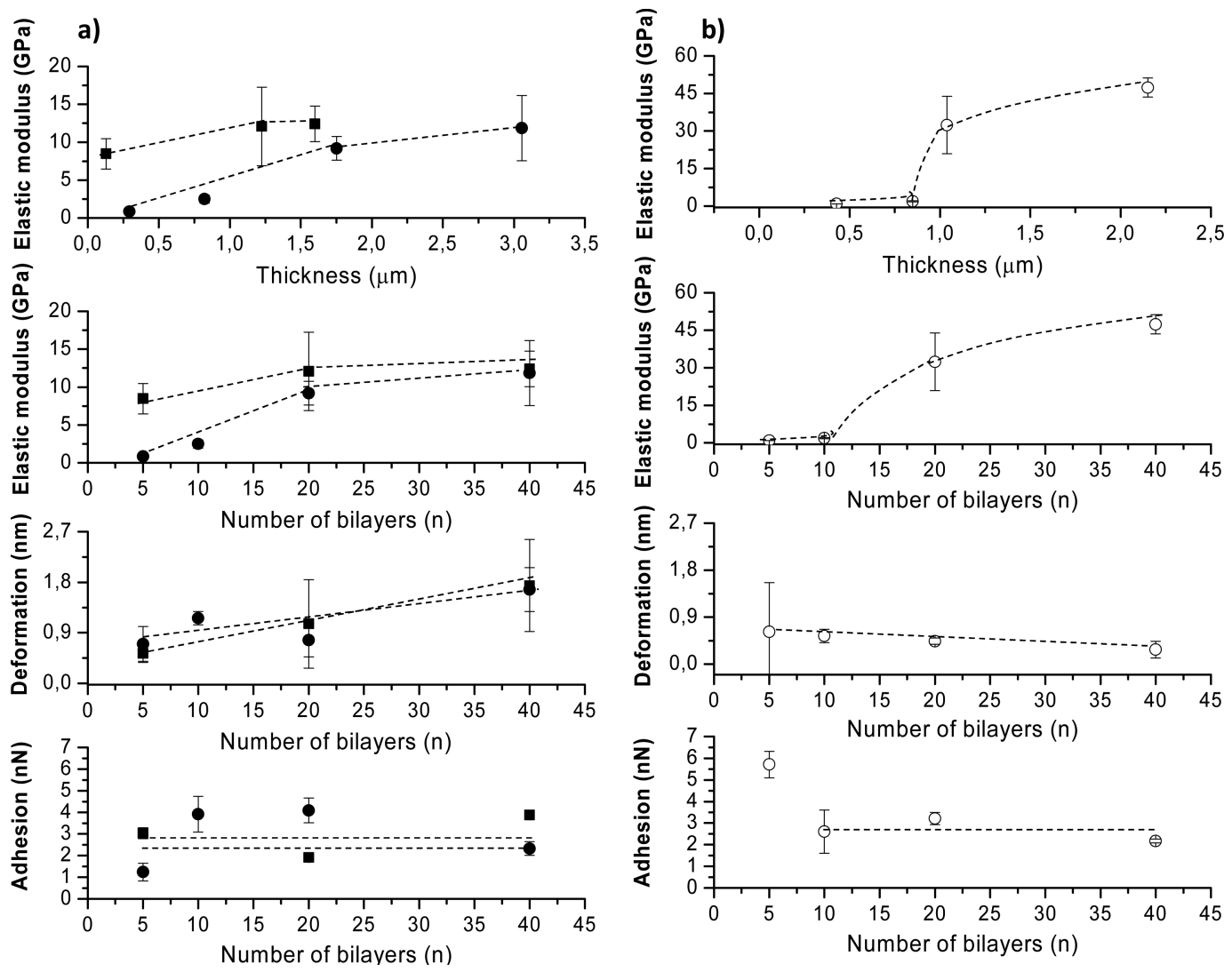

Figure 5. Evolution of the elastic modulus, deformation and adhesion of (a) (Alg1/Chi $)_{n}(\mathbf{\square})$ and $(\operatorname{Alg} 2.5 / \mathrm{Chi})_{n}$ films $(\mathbf{\bullet})$ and (b) cross-linked $(\mathrm{Alg} 2.5 / \mathrm{Chi})_{n}$ films $(\mathrm{O})$ vs film thickness and number of bilayers. Dashed lines are a guide to the eye.
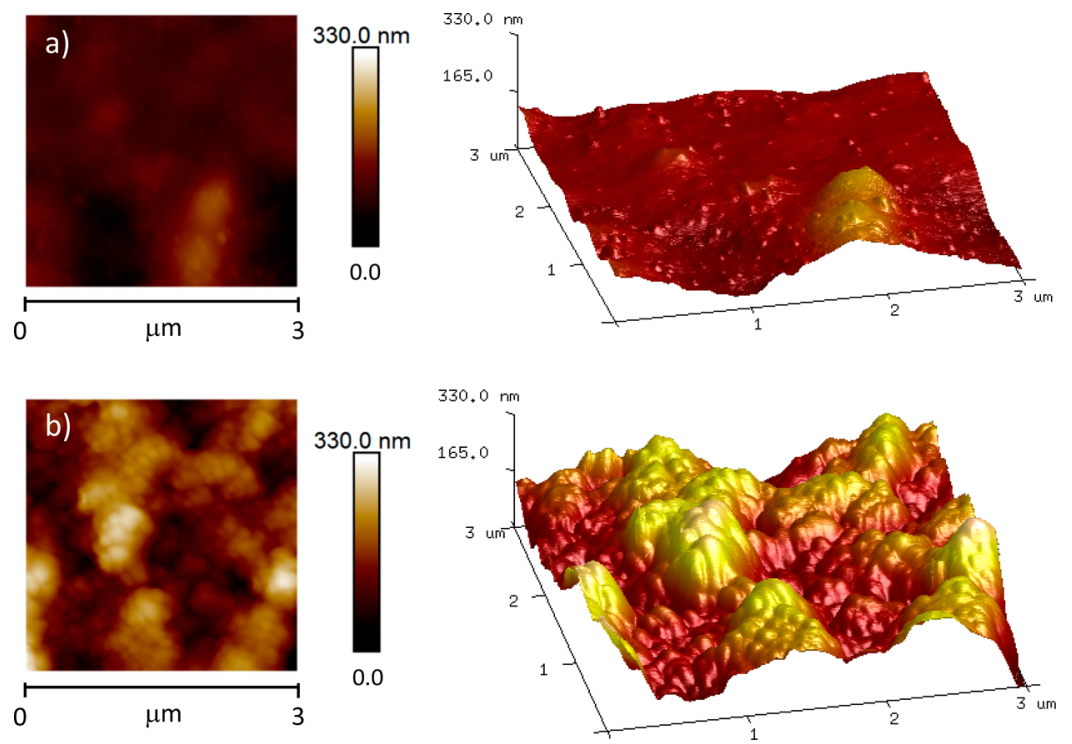

Figure 6. AFM topographic images $(3 \mu \mathrm{m} \times 3 \mu \mathrm{m})$ corresponding to (a) $(\mathrm{Alg} 2.5 / \mathrm{Chi})_{10}$ and (b) nanocomposite $(\mathrm{Alg} / \mathrm{Chi})_{5} /(\mathrm{NPs} / \mathrm{Chi})_{5}$ films.

interpenetration between alginate and chitosan as shown in Figure 3. This result would imply that the adhesion promoted by the final chitosan layer would be higher than that resulting from the interpenetration with alginate. It is important to note that in ambient conditions, the values of adhesion are likely facilitated by capillary forces of a surface adsorbed water layer in air. $^{36}$ This is especially relevant in the case of hydrophilic polymers such as chitosan that constitutes the last layer of all the samples under study. 6,37
To partially summarize, cross-linked films have a higher elastic modulus and less deformation than native films. As expected, cross-linking increases the stiffness of the multilayer film provoking an increase in the elastic modulus. This is due to the fact that thermal cross-linking results in an amidization reaction between the carboxylate groups of alginate $\left(\mathrm{COO}^{-}\right)$ and the ammonium groups of the chitosan $\left(\mathrm{NH}_{3}{ }^{+}\right)$to form amide cross-links (NHCO) that rigidify the multilayers. ${ }^{38}$ The cross-linking reduces the molecular mobility of polymer chains 
because it decreases the intermolecular space between these chains. ${ }^{12}$ The effect of film cross-linking on the elastic moduli was expected since the degree of cross-linking is known to influence the mechanical properties of multilayer films. As an example in a recent study, the Young Modulus of Alg/Chi films, determined on $31.7 \pm 4.7 \mu \mathrm{m}$ film thickness by dynamic mechanical analysis in a hydrated environment at $37{ }^{\circ} \mathrm{C}$, increased from 4.8 to $56.4 \mathrm{MPa}$ after cross-linking with genipin. $^{11}$

Effect of the Incorporation of Nanoparticles on the Nanomechanical Properties. The effect of the incorporation of NPs on the nanomechanical properties of Alg/Chi films was studied. Figure 6 shows representative AFM topographic images corresponding to two samples prepared with the same number of total bilayers, (Alg2.5/Chi) $)_{10}$ and nanocomposite $(\mathrm{Alg} / \mathrm{Chi})_{5} /(\mathrm{NPs} / \mathrm{Chi})_{5}$ films which provide information on their morphology and their roughness. The film without NPs displays finer grain texture and a more homogeneous surface morphology, with an average roughness of $17.1 \pm 3.4 \mathrm{~nm}$, than the nanocomposite film, which exhibits aggregated NPs and an average roughness of $42 \pm 3.4 \mathrm{~nm}$. The formation of aggregates is attributed to the roughness accumulation induced by the inner NPs layers. Cluster formation could be also influenced by the aggregation of small particles during the spray of a colloidal solution. $^{39}$

Representative PF-QNM AFM images corresponding to nanocomposite $(\mathrm{Alg} / \mathrm{Chi})_{10} /(\mathrm{NPs} / \mathrm{Chi})_{10}$ films are shown in Figure 7. The mechanical properties (elastic modulus, deformation and adhesion) were analyzed in four different
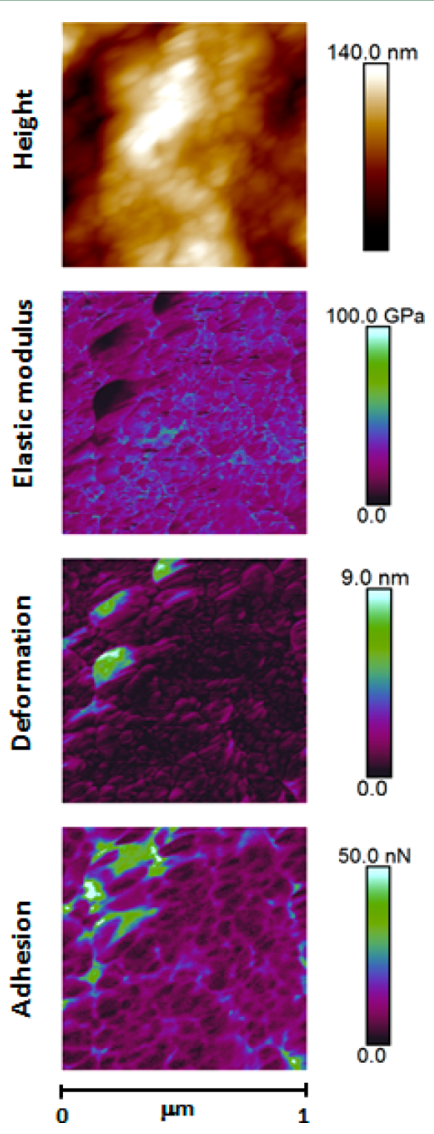

Figure 7. PF-QNM AFM images $(1 \mu \mathrm{m} \times 1 \mu \mathrm{m})$ of height, elastic modulus, deformation, and adhesion corresponding to a nanocomposite $(\mathrm{Alg} / \mathrm{Chi})_{10} /(\mathrm{NPs} / \mathrm{Chi})_{10}$ film. points of these images. It is worth to emphasize that the analysis was carried out in $1 \mu \mathrm{m}^{2}$ regions.

Table 2 summarizes the results corresponding to the thickness determined by SEM and the mechanical properties, determined by PF-QNM AFM, for the three nanocomposite $(\mathrm{Alg} / \mathrm{Chi})_{n} /(\mathrm{NPs} / \mathrm{Chi})_{m}$ films under study.

As it can be observed for the same number of bilayers, nanocomposite films present a higher elastic moduli than $(\mathrm{Alg} 2.5 / \mathrm{Chi})_{n}$ films (results depicted in Figure 5a). These results corroborate that the mechanical properties, found by (PF-QNM) AFM, are consistent with intrinsic properties of the sample and are not influenced by the substrate properties. It is interesting to note that the values of deformation increased significantly in nanocomposite films with respect to $\mathrm{Alg} / \mathrm{Chi}$ films for which the values of deformation range between 0.5 and $1.5 \mathrm{~nm}$. A careful examination of the results depicted in Figure 7 for $(\mathrm{Alg} / \mathrm{Chi})_{10} /(\mathrm{NPs} / \mathrm{Chi})_{10}$ film shows that regions that deform more correspond to regions with no NPs. It might be hypothesized that the incorporation of NPs might decrease the number of interactions between polyelectrolyte layers, thus promoting the formation of "empty regions" in between polymer layers which might lead to the increase of deformation. In fact, previous reports on the incorporation of NPs during the buildup of the multilayer film have revealed that the deposition process can give rise to islands that create empty regions into the internal structure of the film. ${ }^{40,41}$ For the nanocomposite films under study, SEM images shown in Figure 8 corroborate the formation of empty spaces or islands during the spray deposition. The film with a lower number of NPs layers (Figure 8 a) presents separated islands on different points of the surface of the film. As the number of bilayers increases, islands decrease their size and the deposition becomes more homogeneous (Figure $8 \mathrm{~b}$ and $\mathrm{c}$ ).

Furthermore, it is noteworthy that the adhesion value is a factor of 2-3 higher when iron oxide NPs are incorporated within Alg/Chi films (see Table 2) compared to native Alg/Chi films (results depicted in Figure 5), indicating that NPs play a significant role on the adhesion values. As shown in Figure 6, the roughness of nanocomposite films increases with respect to films without nanoparticles. As previously described for samples without nanoparticles, adhesion values are likely to be influenced by the formation of a water neck between the AFM tip and the sample due to capillary condensation and adsorption of thin water films at surface. In addition, surface roughness has also a great influence on the adhesion force measured through AFM although this effect is hard to quantify. ${ }^{36}$ In the case of nanocomposite films, which show higher values of roughness with respect to films without nanoparticles, it might be hypothesized that the formation of empty spaces or islands during the spray deposition might induce an increase in the contact area between the tip and the surface which would have an effect on the increase of the adhesion values found for these samples.

\section{CONCLUSIONS}

We report on the nanomechanical properties of multilayer films made of chitosan (Chi), alginate (Alg), and those obtained by incorporation of iron oxide nanoparticles (NPs) built up via spray assisted layer-by-layer (LbL). To this aim, PeakForce Quantitative Nanomechanical Mapping (PF-QNM) AFM measurements were carried out on supported Alg/Chi multilayer films taking into account three different experimental parameters, namely, alginate concentration, thermal cross- 
Table 2. Summary of Mechanical Properties of Nanocomposite $(\mathrm{Alg} / \mathrm{Chi})_{n} /(\mathrm{NPs} / \mathrm{Chi})_{m}$ Films Determined by PF-QNM AFM and Thickness Determined by SEM

\begin{tabular}{cccc} 
samples & thickness $(\mu \mathrm{m})$ & elastic modulus $(\mathrm{GPa})$ & deformation $(\mathrm{nm})$ \\
$(\mathrm{Alg} / \mathrm{Chi})_{3} /(\mathrm{NPs} / \mathrm{Chi})_{2}$ & $0.24 \pm 0.10$ & $18.8 \pm 6.3$ & $1.1 \pm 0.9$ \\
$(\mathrm{Alg} / \mathrm{Chi})_{5} /(\mathrm{NPs} / \mathrm{Chi})_{5}$ & $0.38 \pm 0.13$ & $34.4 \pm 5.6$ & $1.1 \pm 0.2$ \\
$(\mathrm{Alg} / \mathrm{Chi})_{10} /(\mathrm{NPs} / \mathrm{Chi})_{10}$ & $1.11 \pm 0.34$ & $26.2 \pm 3.9$ & $0.8 \pm 0.2$ \\
\hline
\end{tabular}
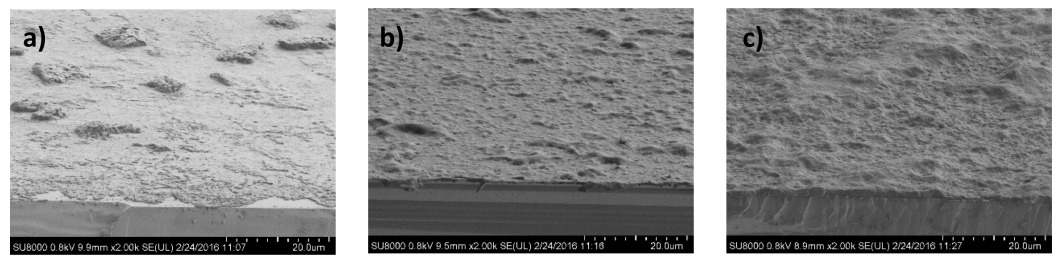

Figure 8. SEM micrographs corresponding to surface of nanocomposite films: (a) (Alg/Chi) ${ }_{3} /(\mathrm{NPs} / \mathrm{Chi})_{2},(\mathrm{~b})(\mathrm{Alg} / \mathrm{Chi})_{5} /(\mathrm{NPs} / \mathrm{Chi})_{5}$, and $(\mathrm{c})$ $(\mathrm{Alg} / \mathrm{Chi})_{10} /(\mathrm{NPs} / \mathrm{Chi})_{10}$.

linking and number of NPs layers. The thickness of Alg/Chi films increased exponentially with the number of layers up to 20 bilayers and then slightly increases toward a plateau. This observation strongly suggests the diffusion of one of the polyelectrolytes "in" and "out" of the film in the range between 5 and 20 bilayers which corresponds to thicknesses in the range of $294 \mathrm{~nm}$ to $1.75 \mu \mathrm{m}$ for Alg2.5/Chi films and $130 \mathrm{~nm}$ to 1.23 $\mu \mathrm{m}$ for Alg1/Chi films. This result is directly correlated to the increase of elastic moduli found for these materials with the increase in the number of bilayers. Thermal cross-linking of $\mathrm{Alg} / \mathrm{Chi}$ films reduces the roughness of multilayer films and increases the elastic modulus, this increase being much more significant for films obtained with higher alginate concentration. For both series of samples, cross-linked and native Alg/Chi films, deformation values are below $2 \mathrm{~nm}$ and the adhesion force varies between 1 and $4 \mathrm{nN}$ regardless the number of bilayers or the concentration of alginate. This is attributed to the fact that the composition of the outer layer is similar for all the studied samples and the adhesion measurements are sensitive only to the surface. The incorporation of iron oxide NPs within the multilayer films increases their roughness and induces an increase of the elastic moduli of the resulting nanocomposite materials. The increase in the values of deformation found for nanocomposite $\mathrm{Alg} / \mathrm{Chi}$ films with respect to $\mathrm{Alg} / \mathrm{Chi}$ films suggests the presence of empty spaces originated from the fabrication process of the films. Finally, values of adhesion of nanocomposite films are higher than those corresponding to $(\mathrm{Alg} / \mathrm{Chi})_{n}$ films which points to a significant effect of the presence of nanoparticles on the adhesion values found for nanocomposite films.

\section{AUTHOR INFORMATION}

\section{Corresponding Author}

*Tel.: +34 915622 900. Fax: +34.915 644 853. E-mail: rhernandez@ictp.csic.es.

\section{ORCID ${ }^{\circ}$}

Rebeca Hernández: 0000-0001-7332-0134

\section{Notes}

The authors declare no competing financial interest.

\section{ACKNOWLEDGMENTS}

Financial support from MINECO (MAT2014-53437-C2-1-P, MAT2014-59187-R MAT2015-66443-C02-1-R and CTQ201343086-P) is gratefully acknowledged. Rebeca Hernández and
Esther Rebollar thank MEC for a Ramón y Cajal contract. Miryam Criado thanks MEC for a FPU fellowship and Técnicas Reunidas S.A. for financial support in Residencia de estudiantes.

\section{REFERENCES}

(1) Langhe, D.; Ponting, M. In Manufacturing and Novel Applications of Multilayer Polymer Films; William Andrew Publishing: Boston, 2016; pp 1-15.

(2) Wong, H. C.; Higgins, A. M.; Wildes, A. R.; Douglas, J. F.; Cabral, J. T. Adv. Mater. 2013, 25, 985-991.

(3) Wang, L.-m.; Chang, H.; Zhang, H.; Ren, K.-f.; Li, H.; Hu, M.; Li, B.-c.; Martins, M. C. L.; Barbosa, M. A.; Ji, J. J. Mater. Chem. B 2015, 3, $7546-7553$.

(4) Ventrelli, L.; Fujie, T.; Turco, S. D.; Basta, G.; Mazzolai, B.; Mattoli, V. J. Biomed. Mater. Res., Part A 2014, 102, 2652-2661.

(5) Discher, D. E.; Mooney, D. J.; Zandstra, P. W. Science 2009, 324, 1673-1677.

(6) Nogales, A.; Ezquerra, T. A.; Rueda, D. R.; Martinez, F.; Retuert, J. Colloid Polym. Sci. 1997, 275, 419-425.

(7) Rescignano, N.; Hernandez, R.; Lopez, L. D.; Calvillo, I.; Kenny, J. M.; Mijangos, C. Polym. Int. 2016, 65, 921-926.

(8) Fujie, T.; Matsutani, N.; Kinoshita, M.; Okamura, Y.; Saito, A.; Takeoka, S. Adv. Funct. Mater. 2009, 19, 2560-2568.

(9) Caridade, S. G.; Monge, C.; Gilde, F.; Boudou, T.; Mano, J. F.; Picart, C. Biomacromolecules 2013, 14, 1653-1660.

(10) Kashiwagi, K.; Ito, K.; Haniuda, H.; Ohtsubo, S.; Takeoka, S. Invest. Ophthalmol. Visual Sci. 2013, 54, 5629-5637.

(11) Silva, J. M.; Duarte, A. R. C.; Caridade, S. G.; Picart, C.; Reis, R. L.; Mano, J. F. Biomacromolecules 2014, 15, 3817-3826.

(12) Gil, S.; Silva, J. M.; Mano, J. F. ACS Biomater. Sci. Eng. 2015, 1, 1016-1025.

(13) Dokukin, M. E.; Sokolov, I. Langmuir 2012, 28, 16060-16071.

(14) Martínez-Tong, D. E.; Najar, A. S.; Soccio, M.; Nogales, A.; Bitinis, N.; López-Manchado, M. A.; Ezquerra, T. A. Compos. Sci. Technol. 2014, 104, 34-39.

(15) Schön, P.; Bagdi, K.; Molnár, K.; Markus, P.; Pukánszky, B.; Julius Vancso, G. Eur. Polym. J. 2011, 47, 692-698.

(16) Nguyen, H. K.; Fujinami, S.; Nakajima, K. Polymer 2016, 87, 114-122.

(17) Richert, L.; Engler, A. J.; Discher, D. E.; Picart, C. Biomacromolecules 2004, 5, 1908-1916.

(18) Derjaguin, B. V.; Muller, V. M.; Toporov, Y. P. J. Colloid Interface Sci. 1975, 53, 314-326.

(19) Sham, A. Y. W.; Notley, S. M. J. Colloid Interface Sci. 2015, 456, $32-41$.

(20) Fernández, R.; Ocando, C.; Fernandes, S. C. M.; Eceiza, A.; Tercjak, A. Biomacromolecules 2014, 15, 1399-1407.

(21) Criado, M.; Sanz, B.; Goya, G.; Mijangos, C.; Hernández, R. Manuscript in preparation, 2016. 
(22) Signini, R.; Campana Filho, P. S. Polym. Bull. 1999, 42, 159166.

(23) Lavertu, M.; Xia, Z.; Serreqi, A. N.; Berrada, M.; Rodrigues, A.; Wang, D.; Buschmann, M. D.; Gupta, A. J. Pharm. Biomed. Anal. 2003, 32, 1149-1158.

(24) Rinaudo, M.; Milas, M.; Dung, P. L. Int. J. Biol. Macromol. 1993, $15,281-285$.

(25) King, K. Food Hydrocolloids 1994, 8, 83-96.

(26) Criado, M.; Mijangos, C.; Hernández, R. Ferrofluidos acuosos de base polimérica con altos coeficientes de absorción específica y procedimiento de obtención. Patent pending, 2016.

(27) Burke, S. E.; Barrett, C. J. Biomacromolecules 2003, 4, 17731783.

(28) Chyasnavichyus, M.; Young, S. L.; Tsukruk, V. V. Jpn. J. Appl. Phys. 2015, 54, 08LA02.

(29) Alves, N. M.; Picart, C.; Mano, J. F. Macromol. Biosci. 2009, 9, 776-785.

(30) Shenoy, D. B.; Antipov, A. A.; Sukhorukov, G. B.; Möhwald, H. Biomacromolecules 2003, 4, 265-272.

(31) Richert, L.; Lavalle, P.; Payan, E.; Shu, X. Z.; Prestwich, G. D.; Stoltz, J.-F.; Schaaf, P.; Voegel, J.-C.; Picart, C. Langmuir 2004, 20, $448-458$.

(32) Lundin, M.; Blomberg, E.; Tilton, R. D. Langmuir 2010, 26, $3242-3251$.

(33) Porcel, C.; Lavalle, P.; Ball, V.; Decher, G.; Senger, B.; Voegel, J.-C.; Schaaf, P. Langmuir 2006, 22, 4376-4383.

(34) Rydzek, G.; Schaaf, P.; Voegel, J.-C.; Jierry, L.; Boulmedais, F. Soft Matter 2012, 8, 9738-9755.

(35) Luo, H.; Shen, Q.; Ye, F.; Cheng, Y.-F.; Mezgebe, M.; Qin, R.-J. Mater. Sci. Eng., C 2012, 32, 2001-2006.

(36) Butt, H.-J.; Cappella, B.; Kappl, M. Surf. Sci. Rep. 2005, 59 (1), $1-152$.

(37) Stukalov, O.; Murray, C. A.; Jacina, A.; Dutcher, J. R. Rev. Sci. Instrum. 2006, 77 (3), 033704.

(38) Yang, S. Y.; Rubner, M. F. J. Am. Chem. Soc. 2002, 124, 21002101.

(39) Taccola, S.; Desii, A.; Pensabene, V.; Fujie, T.; Saito, A.; Takeoka, S.; Dario, P.; Menciassi, A.; Mattoli, V. Langmuir 2011, 27, 5589-5595.

(40) Huang, H.; Spaepen, F. Acta Mater. 2000, 48, 3261-3269.

(41) He, J.; Kanjanaboos, P.; Frazer, N. L.; Weis, A.; Lin, X.-M.; Jaeger, H. M. Small 2010, 6, 1449-1456. 